\title{
Charcot-Marie-Tooth disease type 1
}

Only a year ago in this journal, Vance ${ }^{1}$ reviewed the current state of knowledge of genetic classification of hereditary motor and sensory neuropathies. He also outlined clearly the problems presented to the geneticist when advising patients with hereditary motor and sensory neuropathies types I and II (Charcot-Marie-Tooth disease).

Linkage studies over the last few years have indicated genetic heterogeneity. Although the major locus is on chromosome 17 just above the centromere, ${ }^{2-5}$ a small number of families show linkage to the Duffy blood group locus and the Fc $\gamma$ RII gene on chromosome $1 .{ }^{6}$ No phenotypic differences between the two types are obvious. As most families encountered in clinical practice are too small to provide conclusive evidence for linkage to either of these loci, this has ruled out a linkage approach for either diagnosis or prenatal diagnosis. Additionally, an X linked form $^{7}$ is now well established, which it is often difficult to rule out on pedigree grounds alone.

The severity of clinical symptoms varies widely in CMT type 1 and clinical signs may be virtually absent. However, low nerve conduction velocities measured electrophysiologically provide a firm diagnostic method and are not age dependent. ${ }^{8}$ Many families would prefer to avoid having these electrical tests.

Sporadic cases of CMT pose particular problems for the genetic counsellor as it is not possible to distinguish between autosomal recessive inheritance or a new mutation of a dominantly inherited gene.

The recent reports from groups in Antwerp and Houston $^{9-11}$ of a DNA duplication, detected by probes for the locus D17S122, in Charcot-Marie-Tooth disease go a long way to resolving these dilemmas for the clinical geneticist, meanwhile posing many new questions with respect to the mechanism by which the duplication arises and how this causes the disease.

\section{CMT1a is caused by a large DNA duplication}

The localisation of the CMTla gene has been refined by members of a collaborative group sponsored by the Muscular Dystrophy Association of America to a $3 \mathrm{cM}$ interval within 17p11.2, using linkage analysis. The groups of van Broeckhoven $^{911}$ and Patel and Lupski ${ }^{10}$ independently found that one of the most closely linked markers (409R3 at locus D17S122), originally isolated by David Barker, shows a duplication completely linked to CMT1a. Probe 409R3 detects a three allele MspI restriction fragment length polymorphism with frequent alleles of $2.8 \mathrm{~kb}$ and $2.7 \mathrm{~kb}$ and a rare allele of $1.9 \mathrm{~kb}$. The duplication can be seen in subjects who are heterozygous for the $2.8 \mathrm{~kb}$ and $2.7 \mathrm{~kb}$ alleles by an easily detectable dosage difference or in some cases by the presence of all three possible alleles, that is, $2.8 \mathrm{~kb}, 2.7 \mathrm{~kb}$, and $1.9 \mathrm{~kb}$. Lupski et al ${ }^{10}$ developed a polymorphism for $409 \mathrm{R} 3$ based on a $(\mathrm{GT}) \mathrm{n}$ repeat sequence. This has the advantage of being more polymorphic and therefore more likely to show three separate alleles and of being detected rapidly by polymerase chain reaction amplification. In all, the two groups found the duplication in 204 subjects from 19 pedigrees and in none of 191 unaffected or unrelated subjects. In the American study ${ }^{10}$ five of the seven families were preselected for linkage to chromosome 17, but the families in the Belgian study were selected using only clinical criteria. MacMillan et al ${ }^{12}$ found the duplication in 10 of 11 families selected only by clinical criteria during a population survey of CMT1 in south Wales and Hallam et al (unpublished data) confirmed the presence of the duplication in seven of seven informative families, all of which had previously shown positive lod scores to chromosome 17 probes. It is clear that the duplication is found in the vast majority of CMT1 families. The exceptional family reported by MacMillan et al ${ }^{11}$ did not differ phenotypically from families carrying the duplication. Detection of the duplication will provide a most valuable diagnostic tool in the many families unsuited for linkage analysis and in subjects unwilling to undergo electrical studies. However, complete confidence is not possible until the reason for the lack of duplication in some families ${ }^{11}$ is understood.

Evidence that the duplication in $17 \mathrm{p} 11.2$ is the disease causing mutation comes from the finding of a de novo duplication in an affected subject who appears to be a new mutation in a family with no previously affected members. ${ }^{9}$ Furthermore, a homozygous and severely affected subject from one family was shown to carry four copies of the D17S122 locus ${ }^{10}$ providing further evidence that the duplication is responsible for the disease and that homozygosity results in more serious disease.

The duplication appears to be very large, probably over $1 \mathrm{Mb}$ and containing probes for the loci D17S125 (VAW412R3) and D17S61 (pEW401). Both of these probes had been shown to be tightly linked to the disease locus. Intriguingly, at least one of the breakpoints appears to be constant as a similar extra $450 \mathrm{~kb}$ SacII band has been found in pulsed field gel electrophoresis analysis in affected subjects from seven families. ${ }^{1011}$ This is presumably a junction fragment covering one of the breakpoints.

The duplications appear to have arisen independently and probably by interchromosomal exchange as different and heterozygous allelic forms are found in different families.

\section{The role of new technologies}

With this discovery, the (GT)n type of polymorphism has come of age. Highly informative polymorphisms based on these or similar simple sequence repeats are likely to be widely used diagnostically in the future and are already being developed for analysis of the dystrophin gene in Duchenne and Becker muscular dystrophies. However, here the (GT)n repeat is not only valuable because of its ease of use but because its high level of polymorphism makes it more likely that a trisomy will be shown.

Lupski et $a l^{10}$ confirmed the presence of the duplication in cells of CMT patients in a most elegant manner using two colour fluorescence in situ hybridisation (FISH). Using a red labelled probe for VAW409 and a green labelled probe for 1516 (a chromosome 17 marker outside the duplication) they found three red spots, two near one of the 1516 sites and one near the other, in interphase nuclei from affected subjects. This practical demonstration of the power of this technology is likely to be appreciated by many diagnostic cytogenetic laboratories. 
The challenge to the diagnostic laboratory

There are many implications to be drawn from these articles for molecular diagnostic laboratories, both those involved in CMT and others.

For those involved in CMT there will be the question of the best method for carrier detection and also the issue of prenatal diagnosis. Many people may feel that there is unlikely to be a great demand for prenatal diagnosis in this condition, but certainly some affected families are likely to request it.

At the moment the most technically straightforward method of detecting the duplication is either by visual assessment of dosage in subjects heterozygous for the MspI polymorphism of $409 R 3$ or by the detection of three alleles of the (GT)n polymorphism. Visual assessment of dosage differences is notoriously unreliable. In this case it is particularly straightforward, because the two alleles are so close together that factors affecting strength of hybridisation, such as efficiency of transfer, are unlikely to differ between the two alleles. One confusing factor is that the band with double dosage can shift between $2.8 \mathrm{~kb}$ and $2.7 \mathrm{~kb}$ within one family. In the case where the duplicated chromosome carries one $2.8 \mathrm{~kb}$ allele and one $2.7 \mathrm{~kb}$ allele the band contributed by the normal chromosome may be either 2.8 or $2.7 \mathrm{~kb}$ in different parts of the pedigree leading to double doses of 2.8 or $2.7 \mathrm{~kb}$ respectively. It seems that pulsed field gels will be generally applicable but will require a laboratory to be set up to make agarose blocks on all CMT patients. It is not known whether pulsed field gels are applicable to chorionic villus samples.

Further evidence that the duplication is extremely large comes from the observation that recombinants can still occur between the disease status and the $409 \mathrm{R} 3$ polymorphism (unpublished data), ${ }^{9}$ the presence or absence of the duplication providing the diagnostic gold standard and meaning that there is a finite error using linkage analysis, even using probes from within the duplication.

In addition to the genuine residual chance of crossing over, the unsuspected presence of a duplication can give rise to most misleading linkage information. ${ }^{910}$ The apparent presence of clusters of crossovers or double crossovers was an important clue to both groups who discovered this mutation. As we have no idea how many other examples of this phenomenon may occur, this is a further caution for both researchers and diagnostic laboratories to keep at the back of their minds.

\section{Conclusion}

Prophetically, Vance ${ }^{1}$ concluded his review by stating that "the inherited group of peroneal atrophies have undergone continual classification as more powerful investigative tools have become available". The recent group of papers describing duplications in CMT type la shows even more clearly the relentless power of the new technologies. In addition, they illustrate how the molecular genetic study of a disease inherited in an apparently straightforward dominant mendelian fashion can show new genetic mechanisms. We await eagerly the unravelling of these mechanisms.

Work in the author's laboratory is generously funded by the Muscular Dystrophy Group of Great Britain and Northern Ireland and the Child Health Research Appeal Trust. I would like to thank Professor Anita Harding for many helpful discussions.

SUE MALCOLM

Molecular Genetics Unit,

Institute of Child Health,

30 Guilford Street,

London WC1N $1 E H$.

1 Vance JM. Hereditary motor and sensory neuropathies. $f$ Med Genet $1991 ; 28: 1-5$.

2 Vance JM, Nicholson GA, Yamaoka LH, et al. Linkage of Charcot-MarieTooth neuropathy type la to chromosome 17. Exp Neurol 1989;104:186-9. 3 Middleton-Price HR, Harding AE, Monteiro CJ, Berciano J, Malcolm S. Linkage of hereditary motor and sensory neuropathy type 1 to the pericentromeric region of chromosome 17. Am 7 Hum Genet 1990;46:92-4.

4 Raeymaekers P, Timmerman V, De Jonghe P, et al. Localization of the mutation in an extended family with Charcot-Marie-Tooth neuropathy (HMSN I). Am f Hum Genet 1989;45:953-8.

5 Patel PI, Franco B, Garcia C, et al. Genetic mapping of the autosomal dominant Charcot-Marie-Tooth disease in a large French Acadian kindred: identification of new linked markers on chromosome 17. Am $\mathcal{J}$ Hum Genet 1990;46:801-9.

6 Lebo RV, Dyck PJ, Chance PF, et al. Charcot-Marie-Tooth locus in Lebo RV, Dyck PJ, Chance PF, et al. Charcot-Marie-Tooth
FCgamma RII gene region. Am $\mathfrak{f}$ Hum Genet 1989;45:148A.

7 Rozear MP, Pericak-Vance MA, Fischbeck K, et al. Hereditary motor and sensory neuropathy, X-linked: a half century follow-up. Neurology 1987;37:1460-5

8 Nicholson GA. Penetrance of the hereditary motor and sensory neuropathy Ia mutation: assessment by nerve conduction studies. Neurology 1991;41:547-52.

9 Raeymaekers P, Timmerman V, Nelis E, et al. Duplication in chromosome 17p11.2 in Charcot-Marie-Tooth neuropathy type la (CMT 1a). Neuromuscular Disorders 1991;1:93-7.

10 Lupski JR, Montes de Oca-Luna R, Slaugenhaupt S, et al. DNA duplication associated with Charcot-Marie-Tooth disease type 1a. Cell 1991; 66:219-32.

11 Raeymaekers P, Timmerman V, Nelis E, et al. Estimation of the size of the chromosome $17 \mathrm{p} 11.2$ duplication in Charcot-Marie-Tooth neuropathy type 1a (CMT1a). $\mathcal{Y}$ Med Genet 1992;29:5-11.

12 MacMillan JC, Upadhyaya M, Harper PS. Charcot-Marie-Tooth disease type 1a (CMT 1a): evidence for trisomy of the region p11.2 of chromosome 17 in south Wales families. $\mathcal{f}$ Med Genet $1992 ; 29: 12-13$. 